\title{
WAF: Uma análise de desempenho e eficácia
}

\section{Everton dos Santos Silva, Lucas Azevedo da Silva, Matheus J. da Paixão Frez, Felipe M. H. de Oliveira Malara, Nilson Mori Lazarin}

${ }^{1}$ Bacharelado em Sistemas de Informação - Centro Federal de Educação Tecnológica Celso Suckow da Fonseca (Cefet/RJ) - Nova Friburgo, RJ - Brazil

nilson.lazarin@cefet-rj.br

\begin{abstract}
This article discusses the performance, use of resources and the use of some open source Web Application Firewall (WAF). The performance will be analyzed for each of the three proposed tools. The purpose of this article is for the reader to be able to understand what a WAF is, what its purpose, and with our results, be capable of choosing which WAF is suitable to his web server. In the end, we made a conclusion on the overall efficiency of the proposed WAFs.
\end{abstract}

Resumo. Este artigo discute o desempenho, consumo de recursos e a utilização de alguns Web Application Firewall (WAF) open source. Será analisado o desempenho para cada uma das três ferramentas propostas. O objetivo deste artigo é que o leitor seja capaz de compreender o que é um WAF, para que serve o mesmo, e que seja capaz de escolher com base em nossos resultados qual WAF se adequa melhor ao seu servidor web. No final tiramos algumas conclusões sobre a eficácia geral dos Wafs propostos.

\section{Introdução}

Atualmente cerca de $25 \%$ das aplicações web tem pelo menos uma falha de segurança de alta severidade, sendo a XSS a mais comum e cerca de $28 \%$ dos aplicativos da web não tem nenhuma proteção de força bruta em suas páginas de login [Acunetix 2020]. Visando proteger as aplicações web, os Web Application Firewall (WAF) monitoram, filtram e bloqueiam pacotes de dados conforme eles trafegam em site ou aplicativo da web, contribuindo para uma segurança das aplicações e servidores.

Muitos invasores executam scanners para identificar vulnerabilidades de segurança em um site ou aplicativo para posteriormente lançarem os ataques apropriados. Nos experimentos foram utilizados três ferramentas: Nikto ${ }^{1}$ um scanner de vulnerabilidades catalogadas no OSVDB (Open Sourced Vulnerability Database); OWASP Zed Attack Proxy $^{2}$ um scanner de código aberto desenvolvido pela OWASP, do tipo proxy man-in-themiddle, atuando entre o navegador do testador e o aplicativo da web alvo, capaz de interceptar, alterar e encaminhar pacotes para o destino; e WPScan ${ }^{3}$ um scanner de caixa preta para vulnerabilidades específicas da plataforma WordPress [Broad and Bindner 2014].

Uma possível barreira de proteção contra invasores é a utilização de um WAF que aplica um conjunto de regras a uma comunicação HTTP e pode ser implantado para proteger um aplicativo da web específico ou conjunto de aplicativos da web, sendo considerado

\footnotetext{
${ }^{1}$ https://cirt.net/Nikto2

${ }^{2}$ https://www.zaproxy.org/

${ }^{3}$ https://wpscan.com/
} 
um proxy reverso [Memon et al. 2018] [OWASP 2020]. Este trabalho apresenta um comparativo entre três WAF. Foram considerados diversos aspectos, dentre eles vale destacar: uso de CPU, uso de memória RAM e escrita de disco durante um ataque. Além disso, comparamos as vulnerabilidades encontradas pelos scanners e a severidade das mesmas contra cada WAF.

\section{Metodologia e resultados}

Neste trabalho foram realizadas duas análises: Uma análise de desempenho onde foram considerados os indicadores de uso de CPU, consumo de memória RAM e escrita em disco, mensurando o consumo em estado normal e sob ataque; E uma análise de eficácia que considerou as descobertas dos scanners de vulnerabilidade apresentados anteriormente.

A escolha dos WAFs a serem analisados foi realizada através das seguintes etapas: $1^{\text {a) }}$ Foram listados todos os repositórios públicos identificados com \#waf no GitHub ${ }^{4}$, 233 repositórios foram encontrados; $2^{a}$ ) Selecionamos apenas as ferramentas tipo WAF, ignorando plugins e outros acessórios, 46 repositórios restaram; $3^{\text {a }}$ ) Escolhemos apenas WAF que são tipo Standalone, ou seja, que são autossuficientes e rodam como serviço, 27 repositórios foram selecionados; 4 ) Selecionamos apenas repositórios com mais de 500 estrelas (marcado como favorito por outro membro da comunidade) e que possuíam atualização recente de pelo menos um ano, restando os repositórios listados na Tabela 1.

\begin{tabular}{|c|c|c|c|c|c|}
\hline Repositório & Licenciamento & Estrelas & Contribuidores & Versões & Última versão \\
\hline https://github.com/SpiderLabs/ModSecurity & Apache 2.0 & 4028 & 59 & 90 & $13 / 01 / 2020$ \\
\hline https://github.com/nbs-system/naxsi & GPL-3.0 & 3645 & 39 & 44 & $17 / 11 / 2020$ \\
\hline https://github.com/Janusec/janusec & GNU AGPLv3 & 744 & 2 & 3 & $21 / 12 / 2020$ \\
\hline
\end{tabular}

Tabela 1. Web Application Firewall analisados

Os ataques foram realizados utilizando o Kali Linux, contra três WAF Standalone que protegem o servidor de hospedagem, um Debian 10 com HTTP Apache Server. Todos os membros do ambiente de testes, apresentado na Figura 1a, são máquinas virtuais com a configuração: CPU de 2 núcleos, 1 GB de RAM, 32 GB de HD.

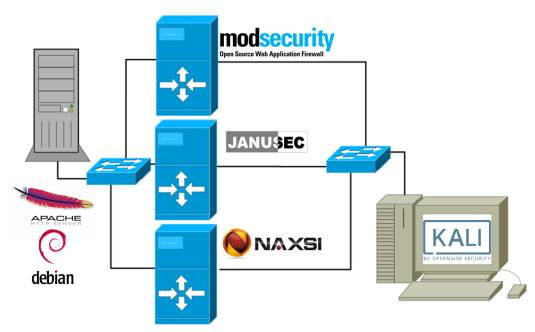

(a) Ambiente

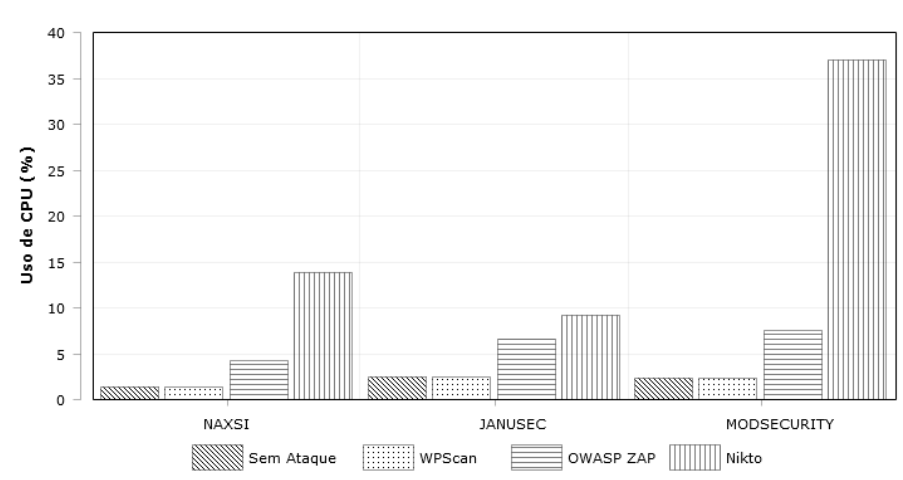

(b) Uso de CPU (\%)

Figura 1. Ambiente de Testes e Consumo de CPU.

\footnotetext{
${ }^{4}$ https://github.com/topics/waf
} 


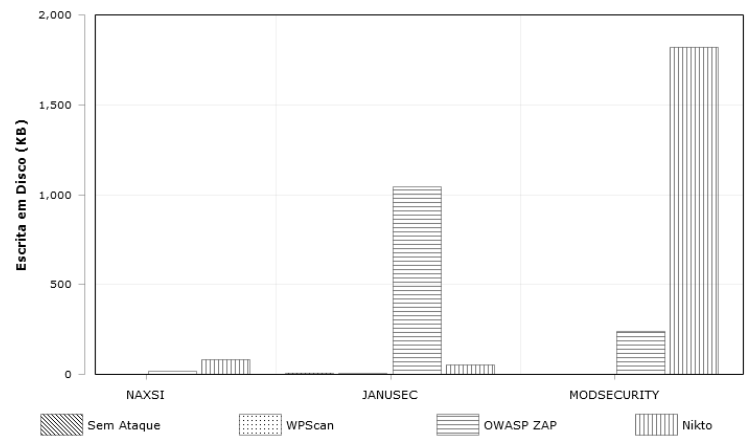

(a) uso Disco (KB)

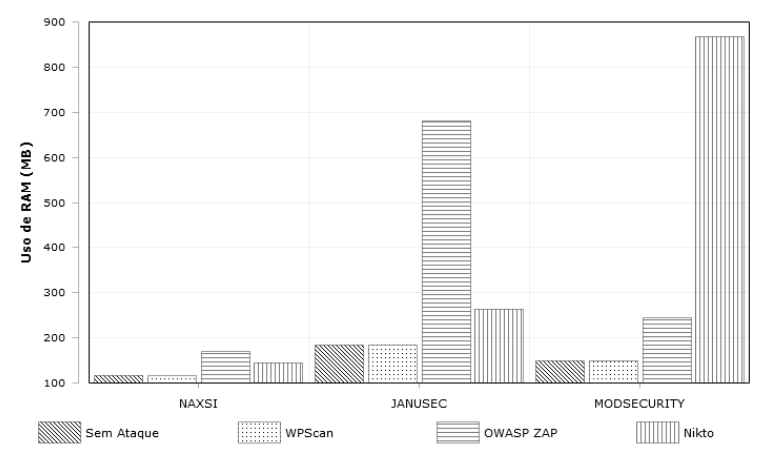

(b) uso RAM (MB)

Figura 2. Consumo de I/O e RAM.

O resultado dos testes de desempenho entre os WAF analisados, em situação normal e sob ataque dos scanners são apresentados em: Figura 1b, percentual de uso de CPU; Figura 2b, consumo em MB da memória RAM; e Figura 2a, escrita no disco em KB.

Durante a análise de eficácia sob ataque do WPScan nenhum plugin foi identificado, foram reportados que o XML-RPC e WP-Cron estão habilitados, e o diretório de uploads está com a listagem habilitada, além dos seguintes resultados: Contra o NAXSI foi possível identificar o readme do Wordpress e a versão utilizada (5.4.4). Foi obtido também a versão e o servidor utilizado no WAF (NGINX); Contra o ModSecurity foi possível identificar a versão e o servidor do WAF (NGINX), o readme e a versão Wordpress, como também o tema da aplicação (twenty-twenty); Contra o Janusec foi obtido o servidor da aplicação (Apache), o readme e a versão do Wordpress.

Os resultados da análise de eficácia sob ataque do OWASP ZAP são apresentados na Tabela 2. Contra o NAXSI foram informados 14 tipos de alerta. Contra o Janusec foram informados 13 tipos de alerta. Contra o ModSecurity foram informados 14 tipos de alerta.

\begin{tabular}{|c|l|c|c|c|c|}
\hline ID & \multicolumn{1}{|c|}{ Alertas (OWASP ZAP) } & Risco & JANUSEC & ModSecurity & NAXSI \\
\hline OR01 & Application Error Disclosure & Médio & $15 \mathrm{x}$ & $6 \mathrm{x}$ & $3 \mathrm{x}$ \\
\hline OR02 & Directory Browsing & Médio & $44 \mathrm{x}$ & $35 \mathrm{x}$ & $50 \mathrm{x}$ \\
\hline OR03 & X-Frame-Options Header Not Set & Médio & $80 \mathrm{x}$ & $76 \mathrm{x}$ & $60 \mathrm{x}$ \\
\hline OR04 & Absence of Anti-CSRF Tokens & Baixo & $3 \mathrm{x}$ & $18 \mathrm{x}$ & $3 \mathrm{x}$ \\
\hline OR05 & Application Error Disclosure & Baixo & $\mathrm{X}$ & $\mathrm{X}$ & $\mathrm{X}$ \\
\hline OR06 & Content-Type Header Missing & Baixo & - & $\mathrm{X}$ & $\mathrm{X}$ \\
\hline OR07 & Cookie No HttpOnly Flag & Baixo & $\mathrm{X}$ & $2 \mathrm{x}$ & $\mathrm{X}$ \\
\hline OR08 & Cookie Without SameSite Attribute & Baixo & $\mathrm{X}$ & $2 \mathrm{x}$ & $\mathrm{X}$ \\
\hline OR09 & Cross-Domain javascript source file inclusion & Baixo & $5 \mathrm{x}$ & $3 \mathrm{x}$ & $5 \mathrm{x}$ \\
\hline OR10 & Private IP Disclosure & Baixo & $2 \mathrm{x}$ & $\mathrm{X}$ & $2 \mathrm{x}$ \\
\hline OR11 & Web Browser XSS Protection Not Enabled & Baixo & $88 \mathrm{x}$ & $87 \mathrm{x}$ & $82 \mathrm{x}$ \\
\hline OR12 & X-Content-Type-Options Header Missing & Baixo & $266 \mathrm{x}$ & $583 \mathrm{x}$ & $303 \mathrm{x}$ \\
\hline OR13 & Information Disclosure - Suspicious Comments & Informativo & $28 \mathrm{x}$ & $36 \mathrm{x}$ & $28 \mathrm{x}$ \\
\hline OR14 & Timestamp Disclosure - Unix & Informativo & $36861 \mathrm{x}$ & $110823 \mathrm{x}$ & $45745 \mathrm{x}$ \\
\hline
\end{tabular}

Tabela 2. Vulnerabilidades e Alertas OWASP ZAP

Os resultados da análise de eficácia sob ataque do Nikto, são apresentados na Tabela 3. Contra o NAXSI foram reportados 17 alertas. Contra o Janusec foram reportados 
8 alertas. Contra o ModSecurity foram reportados 3 alertas.

\begin{tabular}{|c|c|c|c|c|c|}
\hline ID & Vulnerabilidades (Nikto) & Risco & JANUSEC & ModSecurity & NAXSI \\
\hline NR01 & The anti-clickjacking X-Frame-Options header is not present & Baixo & $\mathrm{X}$ & $\mathrm{X}$ & $\mathrm{X}$ \\
\hline NR02 & The X-XSS-Protection header is not defined & Baixo & $\mathrm{X}$ & $\mathrm{X}$ & $\mathrm{X}$ \\
\hline NR03 & The X-Content-Type-Options headers is not set & Baixo & $\mathrm{X}$ & $\mathrm{X}$ & $\mathrm{X}$ \\
\hline NR04 & No CGI directories found & Informativo & $\mathrm{X}$ & & $\mathrm{X}$ \\
\hline NR05 & OSVDB 119 - Web Publisher should be disabled & Médio & $\mathrm{X}$ & & $\mathrm{X}$ \\
\hline NR06 & OSVDB 576 - Weblogic allows source code or directory listing & Baixo & & & $\mathrm{X}$ \\
\hline NR07 & OSVDB 3233 - Apache default file found & Baixo & $\mathrm{X}$ & & $\mathrm{X}$ \\
\hline NR08 & OSVDB 3268 - Directory indexing found & Baixo & $\mathrm{X}$ & & $\mathrm{X}$ \\
\hline NR09 & OSVDB 3288 - Abyss reveals directory listing when are requested & Médio & & & $\mathrm{X}$ \\
\hline
\end{tabular}

Tabela 3. Vulnerabilidades e Alertas Nikto

\section{Conclusão}

Tendo em vista o exposto, podemos perceber que, embora cada WAF tenha sua lógica para lidar com os ataques, em geral, nenhum apresentou vulnerabilidades ou alertas de alta severidade.

O ModSecurity foi o WAF com maior destaque pois, mesmo tendo consumido mais recursos sob ataque, foi o mais eficaz no resguardo das informações, deixando os scanners Nikto e ZAP encontrarem 12 alertas de baixo risco, 3 de médio e 2 informativos, entretanto, foi o único a deixar o WPScan descobrir o tema do WordPress. Em seguida temos o Janusec que teve um uso moderado de recursos durantes os ataques a mesma medida que foi capaz de proteger as informações com 13 alertas de baixo risco, 4 de médio e 3 informativos relatados pelo Nikto e ZAP, além de deixar o WPScan identificar o servidor HTTP. Por último temos o NAXSI que embora tenha sido o WAF que menos utilizou recursos, foi o que mais teve dificuldades na proteção dos dados e teve a maior quantidade de alertas reportados no geral e 15 alertas de baixo risco, 5 de médio e 3 informativos, além de deixar o WPScan identificar a versão do WordPress.

Contudo, para melhor aproveitamento das ferramentas apresentadas é necessário um conhecimento prévio sobre segurança de redes, já que para aplicar as configurações completas são envolvidos alguns conhecimentos avançados de redes. Tendo em vista essa limitação, em trabalhos futuros poderia ser analisado o desempenho dos WAFs de acordo com suas configurações mais avançadas, analisando as melhores configurações para cada tipo de aplicação na web.

\section{Referências}

Acunetix (2020). Acunetix Web Application Vulnerability Report 2020. Disponível em: https://www.acunetix.com/white-papers/acunetix-web-application-vulnerabilityreport-2020/ Acessado em: 15/01/2021.

Broad, J. and Bindner, A. (2014). Hacking com Kali Linux: Técnicas práticas para testes de invasão. Novatec Editora Ltda, São Paulo.

Memon, F., Garrett, O., and Pleshakov, M. (2018). MODSECURITY 3.0 \& NGINX: Quick Start Guide. NGINX, Inc.

OWASP (2020). Web Application Firewall | OWASP. Disponível em: https://owasp.org/www-community/Web_Application_Firewall. Acessado em: $15 / 01 / 2021$. 